\title{
Effect of hemostasis and electrosurgery on the development and evolution of brain tumor surgery in the late 19th and early 20th centuries
}

\author{
John R. Vender, M.D., Jason Miller, B.S., Andy ReKito, M.S., \\ AND DENNIS E. MCDONNELL, M.D.
}

Department of Neurosurgery, Medical College of Georgia, Augusta, Georgia; and The Department of Neurosurgery, Gunderson Lutheran Medical Center, Lacrosse, Wisconsin

\begin{abstract}
Hemostatic options available to the surgeon in the late 19th and early 20th centuries were limited. The surgical ligature was limited in value to the neurological surgeon because of the unique structural composition of brain tissue as well as the approaches and operating angles used in this type of surgery. In this manuscript the authors review the options available and the evolution of surgical hemostatic techniques and electrosurgery in the late 19th and early 20th centuries and the impact of these methods on the surgical management of tumors of the brain and its coverings.
\end{abstract}

KEY WORDS • brain tumor • hemostasis - electrosurgery • history of neurosurgery

The confidence gradually acquired from masterfulness in controlling hemorrhage gives to the surgeon the calm which is so essential for clear thinking and orderly procedure at the operating table.

Halsted

\section{OVERVIEW}

\section{Challenges in Brain Tumor Resection}

The neurological surgeon of the late 1800s and early 1900 s was faced with many challenges in the surgical management of brain tumors. The limited ability to localize these lesions, along with the limited understanding of tumor histological features and biological behavior rendered surgical decision making difficult. Initially, surgeries were targeted only toward lesions with external evidence of either scalp or skull deformity or tumors resulting in easily localized neurological signs. When surgery was attempted, the greatest single hurdle facing the practitioner was the inability to control bleeding. Many of the established surgical practices of the era were ineffective in dealing with the unique tissues and structures of the brain and its blood vessels. Also, the nature of the neurosurgical approach, with its narrow and deep exposures, made use of the ligature nearly impossible for even the most capable surgeon. Initially only surgeries on calvarial or extraaxial lesions were attempted; operations involving penetration of the brain's surface were considered too dangerous. With increasing experience, intraaxial lesions were attempted; however, inability to control hemorrhaging required a fast, crude surgical technique. Surgeons were often forced to "shell out" tumors, usually with a digital or spoon technique, rather than cut into them in an effort to reduce their mass, often resulting in excessive blood loss. With the advent of the electrosurgery unit, tumors could now be debulked internally and the capsule delivered into the cavity. These techniques were already in use for the removal of schwannomas of the vestibulocochlear nerve. These experiences could now be applied to the more vascular lesions.

Originally, access to the intracranial space was obtained through a trephine. Given the limited imaging and localization capabilities of the era, this relatively small exposure (usually $\leq 3 \mathrm{~cm}$ in diameter) required almost perfect placement of the opening. Also, many of the tumors that were diagnosed at the time had reached significant size. Therefore, both because of the tumor size and the surgeon's limited localizing ability, enlargement of the cranial exposure would enhance the effectiveness of the tumor surgery. The disadvantage of large exposures included a longer operating time and increased opening of tissues, both of which resulted in an increase in blood loss. With improved hemostasis, larger cranial exposures were performed and progressively more complex surgeries were attempted.22

One of the most, if not the most, critical additions to the neurosurgeon's armamentarium was the adoption of electrosurgery as a tool for hemostasis, cutting and dissection of tissue, and debulking of tumors. Sachs,$^{21}$ in his manuscript reviewing electrosurgery published in 1931, described three "epoch making contributions to the evolution of neurosurgical hemostasis: the first, Horsley's bone wax, the second, Cushing's silver clip, and the third, the introduction of electrosurgery." In this article we review the hemostatic technologies and technological advances of the era, with an emphasis on the role of electrosurgery. We also discuss the impact of improved hemostatic and dissection 
techniques on advances in the new field of intracranial neuro-oncological surgery.

\section{HEMOSTASIS}

Writing about hemostasis in 1928, Cushing and Bovie ${ }^{9}$ stated the following:

Since the methods of getting in and of getting out of the cranial chamber have become more or less standardized, it is oftentimes easier to expose a tumor than to know just what to do with it when brought to view. The appropriate treatment will depend of course, on many factors which will often put the skill and judgment of the surgeon to a severe test; but the ideal is extirpation of the lesion in one session, and in accomplishing this no technical element is more important than to control the bleeding.

\section{Hemostasis and Brain Tumor Surgery}

Numerous hemostatic options existed for the surgeon at the turn of the 20th century. Unfortunately, the most direct, widely accepted hemostatic technique, vessel ligature, was often not practical in neurosurgery. Although improvements in instrument design helped with the scalp and larger brain blood vessel bleeding, most techniques only indirectly affected blood clotting. Excellent research on the vascular effects of heat and cold irrigation and the use of living tissue grafts was described in this era. Also, by the early part of the 20th century, the clotting cascade was understood and attempts to purify thrombin were underway. ${ }^{18}$ Cautery, a technique that had been in disfavor, was reemerging thanks to the development of new technology and a better understanding of the effects of electrical current on tissue in the early 1800s. This new field of surgical diathermy would dramatically change not only the methods for hemostasis, tissue dissection, and tumor resection, but also the types of tumor surgeries attempted.

Some of the early specialists in brain tumor surgery detailed techniques for control of bleeding on the brain surface. As recounted in Walker's book The Genesis of Neuroscience, ${ }^{22}$ Horsley described spraying a sublimate or saline solution heated to between 110 and $115^{\circ} \mathrm{F}$, followed by the application of gentle pressure with a sponge. Godlee described using galvanic cautery and Roberts applied a hot needle directly to the bleeding vessel.

\section{Specific Hemostatic Concepts}

Position, Pressure, and Patience. As reported in Walker, ${ }^{22}$ the use of gravity to assist with reducing blood loss was widespread. In addition to elevation of the head, when possible, patients were positioned seated or even standing. Numerous techniques evolved for compression of the scalp edges, including rubber bands, tubing, steel rings, plates placed into the wound, and suture. Hemostatic clamps evolved to secure the galea or scalp edges. Dandy developed a curved version of the clamp to allow better folding of the galea and improved compression of the bleeding scalp vessels in a low-profile manner. This technique allows for placement of interrupted galeal closure sutures with the Dandy hemostats still in place. The hemostats are later removed and the sutures tied. This allows for continued hemostasis during scalp closure, which was Dandy's intent. Ultimately, spring-loaded clamps were used for compression of the scalp edges. Mayer first described injecting adrenaline along the incision line. Frazier described surgical exposure and direct compression of the carotid artery in the neck in extreme cases.

Intracranial vessels could be controlled by direct pressure, although this was a time-consuming process dependent on blood pressure and clotting function. Under ideal circumstances clotting will occur in 5 to 8 minutes. Nevertheless, blood pressure fluctuations, further manipulation of the tissue, and removal of the compressive material frequently resulted in rebleeding. Wounds frequently required packing with hot sponges or gauze. This packing could remain in place for several days with the scalp loosely closed, followed by the patient's return to the operating room for removal of the packing. With or without packing in their wounds, many patients were in shock after surgery. New or recurrent bleeding was not uncommon with improvements in blood pressure. Compression of the brain also led to devitalized tissue, necrosis, and increased neurological injury and infection risk..$^{18,22}$

Bone Wax. Bleeding from the diploë created a different type of problem because the bone edges were not compressible and packing was not always reliable. Horsley began to work with bone wax in 1885 . He first reported this use of bone wax based on a technique described by Magendie in the British Medical Journal in 1892. Although successful in dogs in an experimental setting, an antiseptic version needed to be developed for human use. With the aid of Mr. P. W. Squire, an antiseptic compound consisting of beeswax, almond oil, and salicylic acid was developed and successfully used..$^{15,18}$

Styptics. These materials, which have the ability to contract organic tissue and subsequently reduce hemorrhage, never achieved widespread popularity as hemostatic agents. These compounds are mentioned as far back as Galen, who described rules for implanting alum, copper sulfate button, or ferric chloride granules against the vessels of an amputated stump. The styptic is intended to be localized at the point of bleeding and its caustic action involves both the vessel and the neighboring tissue. The downside to this mechanism of action is the formation of an eschar, which covers the bleeding vessel. This precipitation of proteins is not equivalent in specificity and reliability to the crystallization of fibrinogen into fibrin. Other than for use in extreme circumstances, this method was not widely accepted. Nevertheless, in later years, Cushing was reported always to have at hand a basin of Zenker solution, consisting of a corrosive sublimate, potassium bichromate, sodium sulfate, and water. This was used for the destruction of neoplastic remnants after tumor resection and also for application to bleeding spots that failed to respond to other techniques. ${ }^{18}$

The Cushing Clip. In 1911 Cushing introduced a small, implantable metallic clip. ${ }^{7,18}$ These were applied with an instrument similar to modern-day clip appliers. These clips gained widespread use outside the nervous system as well. Initially, this device was used on all vessels; however, after the introduction of electrosurgery, only the larger vessels, which were less amenable to electrosurgical coagulation, were occluded with clips. The original clips were formed by wrapping a silver wire tightly around a rod containing 
Hemostasis, electrosurgery, and the evolution of brain surgery

sharp flutings and grooves on its opposite sides. These several turns of wire were hammered against the flutings to create transverse ridges. The individual clips were then cut apart by scissors sliding along the grooves. These transverse ridges conveyed to the surface of the clips provided additional grip to reduce the risk of the device slipping off the vessel after being squeezed shut.

Hot Irrigation. Although the use of hot-water irrigation to enhance hemostasis was widely known at this time, it appears to have been first presented by Milne Murray, an obstetrician, in a meeting of the Edinburgh Obstetrical Society in 1886 . In the late 1800 s there were significant schools of thought regarding the use of cold as opposed to hot irrigation. Both cold and hot irrigation applied to a blood vessel would result initially in its constriction. This was in contradistinction to the observation that warm irrigation of all degrees would dilate vessels and promote hemorrhage. In experiments conducted in the uterine vascular network of rabbits, Murray showed that the initial contraction seen in both hot and cold irrigation was followed in the case of cold irrigation by an intense compensatory rate of dilation and rehemorrhaging. In the case of hot water-induced vascular constriction, the constriction remained for a longer period of time and ordinary tone was regained slowly with no reactionary vascular congestion. Horsley described the need to obtain water of sufficient temperature to stimulate contraction of small vessels without simultaneously causing heat coagulation of the nervous tissue. His recommendations were that temperatures should be within the 110 to $115^{\circ} \mathrm{F}$ range. This is based on his research findings that water at temperatures ranging from 70 to $105^{\circ} \mathrm{F}$ will dilate blood vessels, whereas from 110 to $115^{\circ} \mathrm{F}$ constriction is noted. In 1911, Cushing advocated a slightly lower temperature range of 100 to $105^{\circ} \mathrm{F} \cdot{ }^{18}$

As Light ${ }^{18}$ recounts, Howell observed that hot irrigation appeared to hasten clotting by accelerating the formation of thrombin. Wiggers also reported that "heat tends to accelerate all chemical reactions concerned in coagulation." Therefore, hot irrigation promotes both temporary vasoconstriction and coagulation of blood. Dusser de Barenne and Zimmerman evaluated the temperatures at which tissue injury would occur based on experiments conducted in the cerebral cortex of monkeys. They determined that an exposure time of 30 seconds to a temperature of $122^{\circ} \mathrm{F}$ resulted in the death of nerve cells in the most superficial cortical layers. Pincus and Fisher, studying the thermal death point of cells in cell culture, believed that a rise of $18^{\circ} \mathrm{F}$ maintained for 30 minutes or less has no apparent effect. They also stated that a temperature increase of $23.4^{\circ} \mathrm{F}$ above normal could be withstood for 2 to 3 minutes and would result in growth inhibition but no cell death. Pontoon concluded that a maximum temperature of $118.6^{\circ} \mathrm{F}$ is conservative.

\section{Live Tissue Grafts and the Clotting Cascade}

Light $^{18}$ also reports that, between 1909 and 1911, Horsley and Cushing simultaneously but separately described how freshly cut grafts of tissue could be used to control venous and capillary bleeding. Although various tissues were effective, muscle was by far superior. Not only was hemostasis obtained but the graft had adequate adhesion to withstand blood pressures of 60 to $80 \mathrm{~mm} \mathrm{Hg}$. Microscopic examination of the region of adherence revealed the presence of blood platelets and fibrin fibrils within a few minutes. Horsley demonstrated that this hemostatic property is lost if the muscle is boiled.

In 1912, Howell identified cephalin as a hemostatic agent. This supplanted previous reports by Wooldridge ${ }^{24}$ describing lecithin. Cephalin was thromboplastic in nature, and Cushing used it to control bleeding from the brain and dura mater by applying it on thin sheets of cotton. When the cotton required removal, however, the clot was disrupted and bleeding resumed. These early observations were the basis for further investigation into the development and purification of compounds for promoting blood clotting as well as the development of degradable, implantable vehicles for their application. ${ }^{18}$

\section{The Rise, Fall, and Rise of Cautery}

The use of heat to seal blood vessels and to control bleeding has been described throughout human history. There are reports of the Egyptians treating tumors with cautery circa $3000 \mathrm{BC}^{3,19}$ Also, in $400 \mathrm{BC}$ Hippocrates described heat as a manifestation as well as a treatment of disease. ${ }^{14} \mathrm{He}$ described the use of cautery specifically for joint problems, such as shoulder dislocations, as well as for hemorrhoids. ${ }^{19}$ From the time of Hippocrates through the Middle Ages, cautery became one of the principal tools of the surgeon. Its simplicity allowed for widespread use. According to Wilkins, ${ }^{23}$ William Harvey once remarked, "Anyone could apply the cautery, practically no one the ligature." The use of the cautery for coagulation was recommended by Albucasis in the 11th century. Over time, however, the use of ligatures became more widespread. In 1552, Paré demonstrated the advantage of the ligature over the cautery. Over the next several hundred years this technique gradually replaced cautery in the control of bleeding. Although sporadically used for many more years, cautery ultimately disappeared from use. It was not until the late 1700 s and early 1800 s that the technological advances of the time made the use of electrically generated heat for the treatment of cutaneous and deep lesions as well as the control of bleeding a treatment option.

\section{The Reemergence of Cautery in Electrosurgery}

Unlike the cautery of the Middle Ages, in the more modern version "no use is made of electrically heated cauteries, wires, or scalpels." With electrosurgery, heat is developed either by the current passing from the active electrode into the tissues, or upon the development of ohmic heat from current passing through the tissues.

Goldwyn $^{11}$ and O'Connor, et al., ${ }^{19}$ described three eras in the chronology of electrotherapeutics. The first was experimentation with static electricity. The second, beginning in 1786, involved galvanization and the induction of muscle spasms by potential differences. The third era began circa 1831 with the induction of specific currents by Faraday and Henry. Vital to the evolution of electrosurgery was the demonstration by Morton in 1881 and d'Arsonval in $1891^{10}$ that sufficiently high alternating frequency can be passed through tissues without producing any physiological effect other than that of heating, unlike the 
physiological stimulation such as muscle twitching and pain known to be associated with lower frequencies of power transmission. Morton originally described a frequency of $100 \mathrm{MHz}$, whereas d'Arsonval discovered that the frequency could safely be lowered to $10 \mathrm{kHz} \cdot{ }^{18,19}$ These observations were the foundation for both medical diathermy and electrosurgery. In both cases the effect is to create heat. With diathermy the heat is diffused over a large area and the rise of temperature is slight and within physiological limits. In contrast, in electrosurgery the current and the heat produced are focused at the end of a small terminal electrode. This results in necrobiotic effects that can range from dehydration to boiling and coagulation of tissue, and even charring of the tissue. Doyen demonstrated that a spark derived from an electrode held close to tissue can produce a temperature in the carbonized area as high as $1100^{\circ} \mathrm{F}$. In comparison, the heated iron used in medieval times was $400^{\circ} \mathrm{F}$ when light yellow, $550^{\circ} \mathrm{F}$ when purple, $1000^{\circ} \mathrm{F}$ when dull red, and $1600^{\circ} \mathrm{F}$ when cherry red. ${ }^{18}$

If the electrode is placed in direct contact with the tissue the concentrated heating effect is lost, and in these circumstances tissue temperatures, measured by Doyen as well as by Ward and West and by Huntoon, ${ }^{16,18}$ reached only 150 to $180^{\circ} \mathrm{F}$. In this manner, the electrosurgical technique could be used within temperature ranges yielding simple protein coagulation. This device, the d'Arsonval apparatus, was initially used for the destruction of skin lesions by Rivière as early as 1900. In 1907, Doyen improved the device by adding a large and indifferent ground plate. By 1910, W. L. Clark had enhanced the power and quality of the electrosurgery instrument by installing multiple spark gaps instead of the single gap that was then in use. Lee deForest in 1908 used a new three-electrode thermionic valve that he had designed, supplying the first radio tube high-frequency apparatus able to provide a cutting current. Experimentation on dogs demonstrated the unit's capability to make clean, precise, and nearly bloodfree incisions. According to Light, ${ }^{18}$ several other reports by Czreny and Dandy revealed that the use of electrosurgery in place of a scalpel was also being performed in Europe at this time. The final refinement of this initial technology was to increase the number of cycles from 500,000 to 2 to 3 million. This was accomplished by Doyen in 1907 to 1908 . The electrical circuit between the patient and the electrosurgery unit is completed by current pass- ing from an active to an indifferent electrode. The amount of heat produced by high-frequency current is proportional to the square of the current density. ${ }^{8,9,13,17,20}$ Density of the current is also critical for the biological effect. High current density is obtained by the use of a small active (operating) electrode with a large inactive (ground or indifferent) electrode.

The diathermy unit was used in general surgery initially, treating lesions of the skin and hemorrhoids as early as 1907, urological lesions including tumors of the bladder by $1910,1,2,4$ and ultimately a variety of conditions, including lesions of the breast, cervix, tongue, and throat. ${ }^{5,6} \mathrm{By}$ 1910 a choice between the multiple spark gap machine and the vacuum tube oscillator was available, the most suitable frequencies had been determined, and a knowledge base regarding the effect of electrical heating on tissue and including the concepts of fulguration, coagulation, desiccation/dehydration, and cutting current had been established (Fig. 1).

\section{Bovie, Cushing, and the Introduction of Electrosurgery to Neurosurgery}

Although available as early as 1910, electrosurgery was not applied to treatment of neurological lesions until 1926. It is difficult to understand the delay in exploring a technical advance that seemed so suited to the unique problems associated with brain and brain tumor hemostasis. Potential negative influences of the day may have delayed adoption of the device. One of the major concerns relating to the use of electrosurgery was the potential, but unknown, immediate and long-term pathological effects from dehydration, coagulation, or charring of tissue or of the coagulated and charred tissue remaining in the brain. Concern was also voiced regarding decreased host resistance and an increased infection rate. Cushing does report an earlier trial with a form of diathermy apparatus that was then on the market. He attempted to make incisions in the brain with an electrified needle and he did not think that this device provided any advantage, either in cutting or hemostasis, over techniques currently in use. His opinion would change, however, when he observed for the first time Bovie's electrical wire loop being used to remove pieces of malignant tissue bloodlessly. ${ }^{8,9}$

In June of 1925, Cushing was attending a meeting of the American Medical Association in Atlantic City, New Jer-

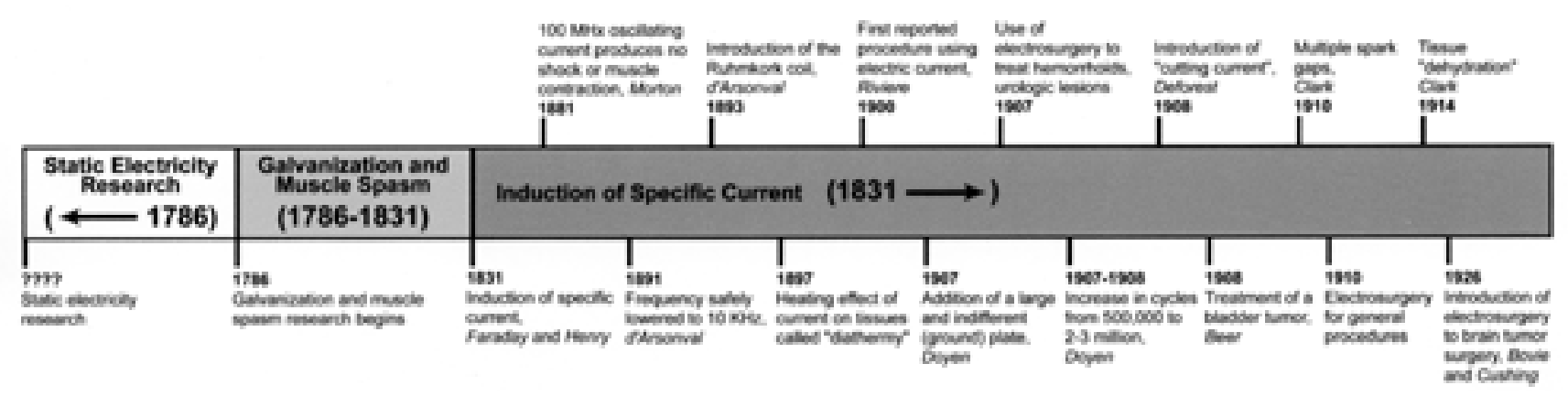

Fig. 1. Timeline showing the three eras of the evolution of electrotherapeutics as well as key events or developments in the evolution of electrosurgery. 
sey, where he met Drs. S. C. Harvey and John Morton. At the time they were watching a demonstration of the use of a desiccating and cutting diathermy machine on a block of beef. It was suggested "in a purely jocular fashion" to Dr. Cushing that he should use this device on the brain. The suggestion was raised in jest, likely due to the crude nature of the procedure. Nevertheless, the idea resonated with Cushing. Although it has been suggested that Cushing may actually have attended that meeting out of interest in the technology, he gave no indication of this. ${ }^{23}$

On Cushing's return to Boston he became aware that W. L. Clark's method of electrosurgery was being used at the Huntington. At that time Dr. William T. Bovie (Fig. 2) was the physicist working on improving the high-frequency apparatus and developing a better device. After discussions between Cushing and Bovie it was agreed that the latter's electrosurgical unit would be used to attempt the removal of a brain tumor. Of particular interest was the ability of the loop cautery to remove "scallops" of tissue bloodlessly. The term "scallop" was a descriptive term adopted to describe the shavings of tissue retrieved with each passing of the loop cautery. The initial unit had a foot pedal; however, Cushing believed that this limited the surgeon's ability to move during the procedure by forcing him to remain in one position. Therefore, a pistol device with a trigger was developed. In this fashion the surgeon

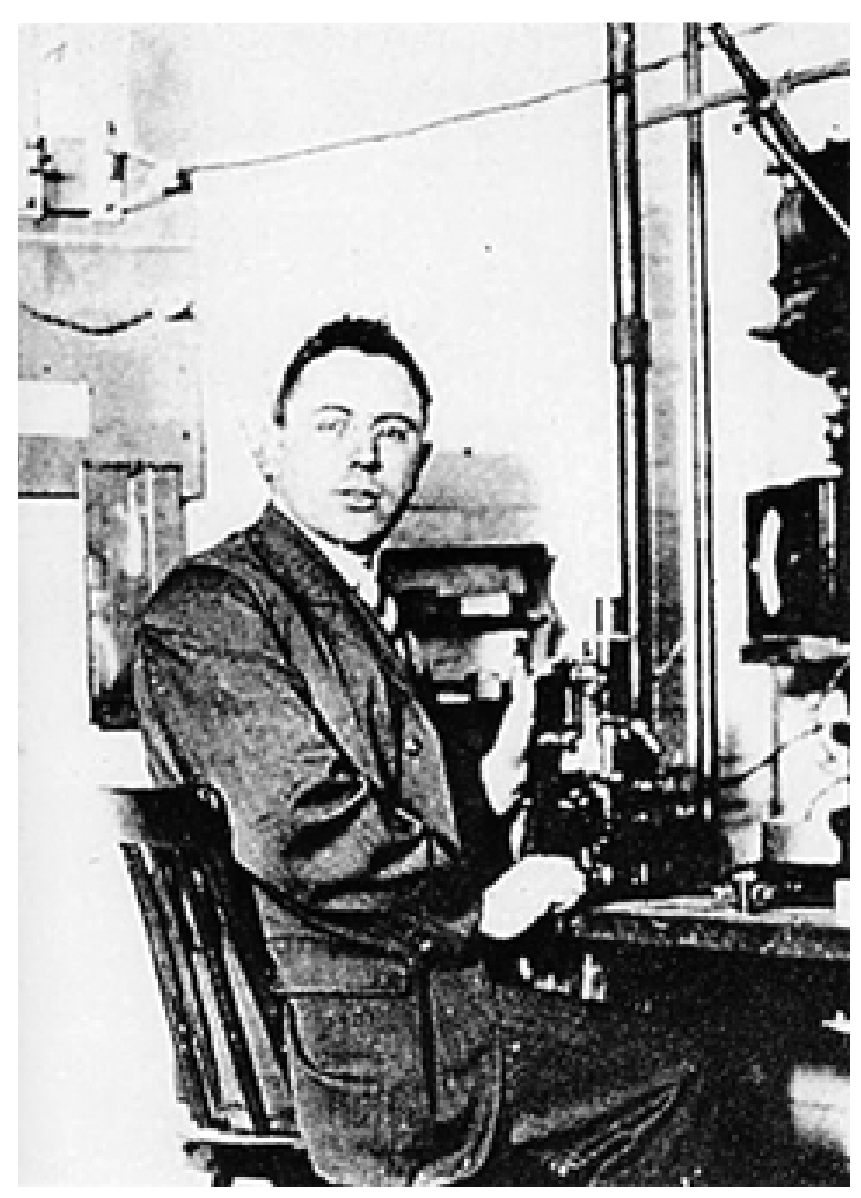

Fig. 2. An early photograph of Dr. William T. Bovie. could activate the device simply by pulling a trigger. ${ }^{9}$ Multiple styles of operating electrodes were created and could be used interchangeably with the pistol grip.

There are three distinct ways in which various high-frequency currents delivered by the electrosurgery unit can be applied in this modality. These include superficial dehydration, cutting, and tissue coagulation. To achieve superficial dehydration, the active electrode is held close to but not actually in contact with the tissue to be dehydrated. In this manner the sparks are "sprayed" across the intervening space. Due to the high temperature of the spark, sufficient heat is generated to dehydrate completely a thin superficial layer of tissue. For it to serve as a cutting tool, a different type of current is passed through the active electrode. The cutting is performed not by the electrode itself but by the current forming ahead of the electrode in an electrical arc. This volatilizes and separates the tissues. When cutting tissue in this fashion, a modification in the current (due to a variable amount of damping of the current) can create either a greater or lesser degree of coagulation or dehydration on the edges of the cut tissues. This can be adjusted based on the vascularity of the tissues being cut. With less vascular tissues, current producing a more pure cutting effect is used, whereas more vascular tissues are dehydrated or coagulated while being cut to enhance the hemostatic effect. Also, when cutting, a strong current can make deep incisions rapidly, whereas weaker currents are preferable for more delicate, bloodless dissections. For coagulation (electrocoagulation) of tissue, a different type of current was used. In this case the active electrode cannot pass through the tissue. Nevertheless, the tissue surrounding the electrode becomes heated to a depth that depends on both the density of the current and the length of time the current is permitted to flow. For coagulation of large tissue masses, a weak current can be used for a prolonged period of time until the tissue becomes heated. This is in preference to a current that would immediately coagulate the tissue. With smaller tissue volumes requiring coagulation, a strong current can be applied for a shorter period of time, thereby preventing any ill effects to the surrounding tissues. ${ }^{9}$ The electrosurgical unit has switches that allow the active electrode to be energized with the particular kind of current required by the surgeon. These controls are operated by an assistant according to the surgeon's instruction (Fig. 3).

The first patient to undergo electrosurgically assisted tumor resection is described as a 64-year-old paper maker with a history of swelling on the right side of his head that began 5 months after recovering from an episode of pneumonia. The lesion had slowly progressed in size, and x-ray films demonstrated defective and highly vascularized bone underlying the tumor. At the time, the leading diagnosis was a rapidly growing sarcoma. An initial procedure was attempted to remove the extracranial portion of the growth by scraping it from the underlying skull bone. Excessive bleeding resulted in the procedure being aborted and hemostasis being restored using established techniques of muscle and cotton pledgets soaked with Zenker fluid. A second stage of the procedure was performed several days later with the Bovie unit. Cushing described the session as follows: "with a damped, dehydrating current the remaining extracranial portion of the growth was removed with surprising ease by the loop electrode and 

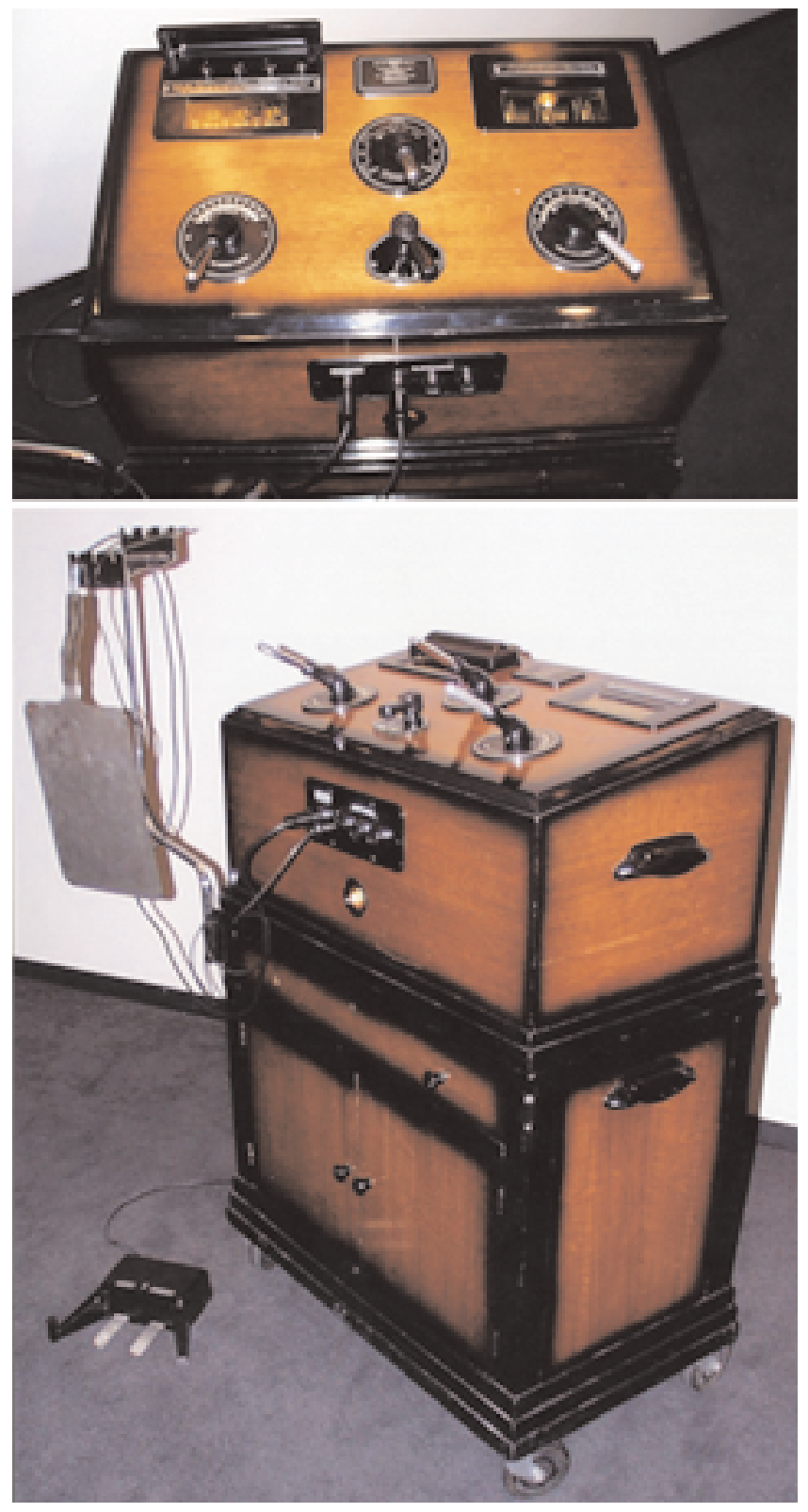

Fig. 3. Photographs showing (upper) top and (lower) lateral views of an early version of the Bovie electrosurgical unit used as late as the 1950s. Of note, in the lower photograph, the large plate is positioned by the wall in the background.

bleeding points checked by coagulation." 9 The next step of the procedure was to create and perform a series of perforations connected by a Montenovesi forceps. The flap was closed due to profuse bleeding. Ten days later, during the third stage of the procedure, the entire block of bone and dura mater was removed. There was no evidence of any unusual reactions from the very large region of charred tissue that was coagulated during the second stage of the operation. The electrosurgery unit and clips were used to control dural bleeding. The pathological diagnosis was a myeloma of plasma cell type. Cushing conceded in his manuscript that with his later knowledge of electro- surgery the procedure would most likely have been done in one sitting. ${ }^{9}$

Evolution of the original electrosurgical unit resulted in the fabrication of a "pencil" type handle. This was required, given the increasing complexity and delicate nature of tumor surgeries that were being performed. The pistol-shaped unit was difficult for the surgeon to control during these more precise maneuvers. Therefore, the use of the foot pedal was reinstated for this new handle. ${ }^{9}$

Ultimately, Cushing described the removal of more than 500 tumors during the first several years of using the electrosurgery unit. This not only included new patients, many of whom had tumors previously considered inoperable, but also earlier patients whose lesions had been previously deemed inoperable but who were called back for an attempted surgery with this new technology.

The early success of the procedure and the publicity from Cushing's writings helped electrosurgery rapidly gain widespread use in neurosurgery. The electrosurgical unit, although it has evolved from its earliest type, remains fundamentally unchanged..$^{21}$ Arguably, only the bipolar device introduced by Greenwood in $1940^{12}$ represents a fundamental change. Ironically, although the electrosurgical monopolar unit still bears his name, Bovie never benefited from his device; he had sold his patent to the Liebel-Flarsheim company of Cincinnati for $\$ 1{ }^{19}$

\section{Early Complications of Electrosurgery}

A key initial concern regarding the use of electrosurgery in the nervous system was infection. Although there were several infections in Cushing's original series, he ultimately attributed these to faulty technique rather than any lowering of the resistance of host tissues. The early electrosurgery unit, unlike modern models, required several operators to be present in the operating room to turn switches and make adjustments in the current. These additional personnel, many of whom were unfamiliar with surgical procedures and sterile technique, may have contributed in the early years to the occurrence of some infections. ${ }^{9}$

With the initial technology, over the course of long operations, patients could become electrically charged to the point where a spark would result if the anesthesiologist contacted the patient's face. There was concern therefore regarding the possibility of an ether explosion or fire. Cushing reports only one episode in which ether vapor was ignited. This was during a frontal surgical procedure in which a small hole was made in the frontal sinus during the course of the operation. This resulted in a direct communication between the respiratory passages and the surgical field. Although the ether vapor did ignite, there were no reports of any injuries. ${ }^{9}$

Cushing also reported several epileptiform seizures during the use of the electrosurgery unit. Although the high alternating currents themselves had no stimulating effect on the tissue, it was postulated that diffusion of the current from the point of discharge was capable of producing this physiological response. ${ }^{9}$

There was great concern about electric shocks and burns, to the patient as well as to the surgeon and assistant, both of which were encountered early in the use of the electrosurgery unit. Initially a wooden operating table and 
wooden spatula were adopted due to these early experiences. With refinements in the electrosurgery apparatus and increasing experience with electrosurgery techniques, however, such modifications were no longer required. ${ }^{9}$

\section{CONCLUSIONS}

The brain tumor surgeon of the late 19th and early 20th centuries encountered numerous obstacles when treating patients with intracranial neoplasms. Limited localizing technology and limited histopathological understanding of the tumor's behavior were compounded by the edema often encountered in the brain and the difficulty controlling bleeding. Of these various factors, massive blood loss was repeatedly cited as the number one limitation to the development of better surgical treatment. The introduction of the electrosurgical unit contributed to the provision of larger and more extensive exposures; allowed for longer operating times in which greater care could be taken to identify, dissect, and remove tumors; and led to a reduction in infection rates and anesthesia risks by reducing the number of staged procedures and the number of procedures that required packing. Most of our modern hemostatic tools, which we rely on daily to enhance the safety of our brain tumor surgeries, have their genesis in this era. Hemostatic agents targeting the clotting cascade, absorbable implants, bone wax, surgical clips, surgical hemostatic instruments, and electrosurgery (both monopolar and, several decades later, bipolar techniques), although they have evolved, are fundamentally similar to their predecessors. The improved potential of the tumor surgeon drove the need for these technologies and they, in turn, helped advance the field of brain tumor surgery.

\section{References}

1. Beer E: On the value of cauterization by the high frequency current in certain cases of prostrate obstruction. NY Med J 98: 170-172, 1913

2. Beer E: Removal of neoplasms of the urinary bladder. A new method, employing high-frequency (Oudin) currents through a catheterizing cystoscope. JAMA 54:1768-1769, 1910

3. Breasted JH: The Edwin Smith Surgical Papyrus. Chicago: Chicago University Press, 1930, p 54

4. Bugbee HG: The use of the high frequency spark in the treatment of median bar obstruction. Urol Cutaneous Rev 7: 361-364, 1917 (Reference unverified)

5. Clark WL: The desiccation treatment of congenital and new growths of the skin and mucous membranes. JAMA 63: 925-928, 1914
6. Clark WL: Oscillatory desiccation in the treatment of accessible malignant growths and minor surgical conditions. A new electrical effect. J Adv Ther 29:169-180, 1911

7. Cushing $\mathrm{H}$ : The control of bleeding in operations for brain tumors. With the description of silver "clips" for the occlusion of vessels inaccessible to the ligature. Trans Am Surg Assoc 29:389-410, 1911

8. Cushing H: The Meningiomas Arising From the Olfactory Groove and Their Removal by the Aid of Electro-surgery. Glasgow: Wylie, 1927, p 53

9. Cushing H, Bovie WT: Electro-surgery as an aid to the removal of intracranial tumors. With a preliminary note on a new surgical-current generator by W. T. Bovie. Surg Gynecol Obstet 47:751-785, 1928

10. d'Arsonval A: Action physiologique des courants alternatifs. $\mathbf{C}$ R Soc Biol 43:283-286, 1891

11. Goldwyn RM: Bovie: the man and the machine. Ann Plast Surg 2:135-153, 1979

12. Greenwood J Jr: Two point coagulation. A new principle and instrument for applying coagulation current in neurosurgery. Am J Surg 50:267-270, 1940

13. Harvey SC: The History of Hemostasis. New York: Haeber, 1929, p xvi, 128

14. Hippocrates: The Genuine Works of Hippocrates (translated by Adams F). London: London Sydenham Society, 1849, p 827

15. Horsley V: Antiseptic wax. Br Med J 1:1165, 1892

16. Huntoon RD: Tissue heating accompanying electrosurgery. An experimental investigation. Ann Surg 105:270-290, 1937

17. Kelly HA, Ward GE: Electrosurgery. Philadelphia: WB Saunders, 1932, p 305

18. Light RU: Hemostasis in neurosurgery. J Neurosurg 2: 414-434, 1945

19. O'Connor JL, Bloom DA: William T. Bovie and electrosurgery. Surgery 119:390-396, 1996

20. Pearce JA: Electrosurgery. New York: Wiley \& Sons, 1986, pp 25-76

21. Sachs E: Electrosurgical unit as an aid to the neurologic surgeon. Surg Gynecol Obstet 52:505-507, 1931

22. Walker AE: The Genesis of Neuroscience. Chicago: Chicago University Press, 1998, pp 268-273

23. Wilkins RH: Neurosurgical classic. XXXV. J Neurosurg 23: 85-116, 1965

24. Wooldridge LC: On the coagulation of the blood. J Physiol 5: xi-xii, 1884

Manuscript received February 15, 2005.

Accepted in final form March 14, 2005.

Address reprint requests to: John R. Vender, M.D., Department of Neurosurgery, BI 3088, 1120 15th Street, Augusta, Georgia 30912. email: jvender@mail.mcg.edu. 